\title{
Pérou: Informer les clients sur le mode d'utilisation de leur méthode de choix
}

Frontiers in Reproductive Health

Follow this and additional works at: https://knowledgecommons.popcouncil.org/departments_sbsr-rh

Part of the International Public Health Commons, and the Women's Health Commons How does access to this work benefit you? Let us know!

\section{Recommended Citation}

"Pérou: Informer les clients sur le mode d'utilisation de leur méthode de choix," FRONTIERES Résumés de Recherche Opérationnelle. Dakar: Population Council, 2000. 


\section{Pérou Informer les clients sur le mode d'utilisation Qualité des Soins de leur méthode de choix}

\section{$N^{\circ} 13$}

Pour une gestion plus efficace du temps imparti au counseling, les prestataires des services de planification familiale au Pérou doivent s'employer davantage à fournir aux clientes des informations utiles et à leur poser des questions pertinentes sur les méthodes qu'elles ont choisis.

\section{Contexte}

En 1998, le Ministère de la Santé (MS) du

Pérou a mis au point des normes de qualité de soins pour s'assurer que les prestataires des services planification familiale (PF)

fournissent à leurs clientes des informations qui correspondent à leurs besoins et objectifs. En 1999, le Population Council, en collaboration avec le MS, a mené une étude pour déterminer si la durée des séances de counseling avait un impact sur le volume d'informations fournies aux clientes.

Cette étude sur la qualité des soins fournis a porté sur 19 centres de santé tirés au sort dans 10 quartiers urbains sur un total national de 172 centres. Six clientes simulées (choisies à l'occasion comme clientes) ont effectué 114 visites dans 19 centres de santé de juin à juillet 1999. Chacune des clientes simulées avait suivi une orientation pour dire qu'elle voulait passer de la méthode des températures à une méthode plus efficace. Après le counseling, elle devrait choisir le Depo-Provera injectable, mais en disant qu'elle souhaitait consulter son mari avant de commencer à l'utiliser.

Pour évaluer la qualité du counseling, les clientes ont complété un formulaire de vérification fiche de contrôle après chaque visite pour indiquer les informations qui leur avaient été données. Le formulaire de vérification de fiche de contrôle comprenait 46 points basés sur un seuil maximum d'informations à échanger. Elle a porté sur des questions d'identification des facteurs relatifs aux options contraceptives Elle a porté sur des questions d'identification des facteurs relatifs aux options contraceptives ; des informations sur les méthodes de

contraception disponibles; des question sur les contre-indications de l'utilisation de l'injectables ; les informations sur l'utilisation de l'injectable ; les effets secondaires et les précautions d'emploi ; l'utilisation des méthodes de barrière pour une protection temporaire contre une grossesse ; et les instructions de suivi.

\section{Résultats}

Comparées aux séances plus courtes, les séances de counseling qui durent $9 \mathrm{mn}$ ou plus permettent aux agents de communiquer un volume d'informations plus important. Pendant les séances allant de 9 à 14 minutes, les prestataires ont couvert 42 pourcent des 46 éléments sur la fiche des informations à échanger, contre seulement 30 pourcent pendant les séances plus courtes de 8 minutes ou moins. Toutefois, les échanges d'informations n'ont connu qu'une légère amélioration pendant les séances allant de 15 à 45 minutes.

\section{Echanges d'informations durant la séance de conseling}

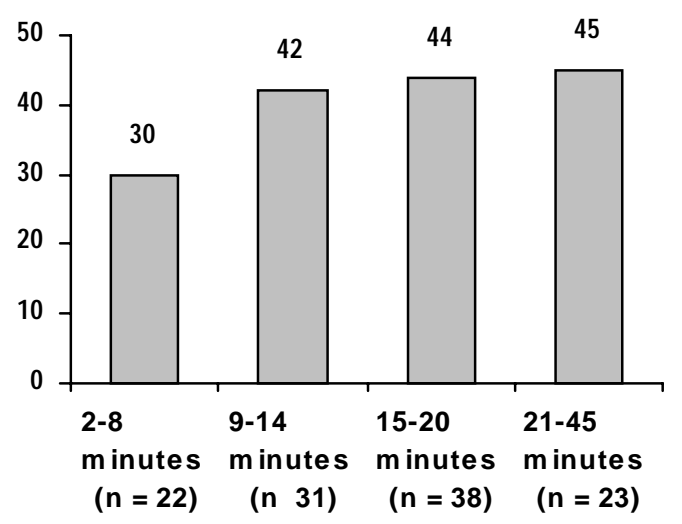

$\square$ Durée de la séance de counseling 
- Les séances de counseling plus longues n'ont pas abouti à plus d'informations parce que les prestataires ont utilisé le temps supplémentaire à fournir aux clientes des détails sur les méthodes qu'elles n'ont pas l'intention d'utiliser au lieu de leur donner l'information essentielle sur les méthodes qu'elles ont choisi et ses contre-indications. Dans moins de 20 pourcent de visites, les prestataires ont posé des questions relatives aux saignées vaginales, discuté du cancer du sein, conseillé dans la période intérimaire l'utilisation des méthodes de barrière ou demandé si la cliente a compris.

Néanmoins, les prestataires ont pu atteindre plusieurs objectifs fixés sur la qualité de soins. Dans plus de quatre sur cinq séances de counseling, les prestataires ont posé les questions standards permettant d'évaluer les options contraceptives appropriées, présenter 4 méthodes de contraception ou plus, et demander à la cliente de choisir une méthode de contraception.

Les prestataires impliqués dans cette étude ont accueilli favorablement la retro-information sur la qualité de leur counseling. Ils ont cité les contraintes de temps comme la cause majeure d'insuffisance du counseling. Certaines visites étaient nettement trop courte, mais la durée moyenne des visites était de 15 minutes, ce qui aurait dû permettre une couverture suffisante des informations clés. Les prestataires ont aussi raté les chances d'offrir à chaque cliente un traitement approprié en lui posant des questions pour vérifier sa situation et ses besoins.

\section{Implications pour les programmes}

- Le MS doit tester une méthode alternative de counseling pour aider les prestataires à offrir aux clientes des conseils plus efficaces et mieux appropriés à leurs besoins en matière de planification familiale. Ce modèle comprend cinq étapes :

1. Accueil chaleureux ;

2. Diagnostic axé sur les besoins de la cliente et permettant d'identifier aussi des méthodes appropriées de planification familiale ;

3. Offre d'informations appropriées et personnalisées sur les méthodes appropriées, permettant de choisir une seule méthode ;

4. Examen des contre-indications, la formation sur l'utilisation de la méthode choisie, et la mise à disposition des consignes de suivi ;

5. Retro-d'informations pour assurer une bonne compréhension et un suivi approprié.

Ce modèle met l'accent sur le rôle que doit jouer le prestataire après le choix de la méthode de contraception, y compris l'examen des contre-indications, les explications sur l'utilisation correcte de cette méthode, la discussion des effets secondaires et des précautions d'emploi. Le MS doit développer des manuels pour aider les prestataires à mettre en pratique ce modèle et entreprendre une recherche opérationnelle pour en évaluer son impact.

Septembre 2000

Léon, Federico R. et al., 1999. « Durée des Séances de Counseling et taux d'échange d'informations dans les Cliniques Péruviennes ». Pour plus d'information, contacter : Population Counncil, Av. San borja Sur 676, Lima 43, Peru. Té. : 511-475-0275 ; Fax : 511 475-0675; E-mail : pclima@amauta.rcp.net.pe ou contacter : population Council, Escondida 110, Villa Coyoacàn, 04000, Mexico, D. F. Mexico.Tél. : 52-5659-8537; Fax : 52-5554-1226 ; E-mail : disemina@ popcouncil.org.mx.

Ce projet a été réalisé avec le concours de L'AGENCE AMERICAINE POUR LE DEVELOPPEMENT INTERNATIONAL (USAID) aux termes du contrat numéro CCP-3030-C-00-3008 et de l'accord de coopération numéro HRN-A-00-98-00012-00.

\section{(1) Population Coundal}

